\title{
Cluster relaxation dynamics in liquids and solids near the glass-transformation temperature
}

\author{
V.B. Kokshenev \\ Departamento de Física, Universidade Federal de Minas Gerais, Instituto de Ciências Exatas \\ Caixa Postal 702, Belo Horizonte 30123-970, Brazil \\ E-mail: valery@fisica.ufmg.br; valery.kokshenev@gmail.com
}

Received October 5, 2006

\begin{abstract}
The structural relaxation in glass forming materials is studied near the glass transformation temperature $T_{g}$ indicated by the heat capacity maximum. The late-time asymptote of the Kohlrausch-Williams-Watts form of the relaxation function is rationalized via the mesoscopic-scale correlated regions in terms of the Debye-type clusters following the dynamic scaling law. It is repeatedly shown that regardless of underlying microscopic realizations in glass formers with site disorder the structural relaxation is driven by local random fields, described via the directed random walks model. The relaxation space dimension $d_{S}=3$ at $T_{g}$ is suggested for relaxing units of fractal dimension $d_{f}=5 / 2$ for quadrupolar-glass clusters in ortho-para hydrogen mixtures, that is compared with entangled-chain clusters in polymers $\left(d_{f}=1\right)$ and solid-like clusters relaxing in supercooled molecular liquids (with $d_{s}=6$ and $d_{f}=3$ ). The relaxation dynamics of orientational-glass clusters in plastic crystals is attributed to the model of continuos time random walks in space $d_{s}=6$. As a by-product, the expansivity in polymers, molecular liquids and networks is predicted.
\end{abstract}

PACS: 61.41.+e Polymers, elastomers, and plastics;

61.43.Fs Glasses;

64.70.Rf Glass transitions.

Keywords: cluster, relaxation, fitting forms, fractal cluster, fractal cluster treatment, thermodynamic instability.

\section{Introduction}

The process of structural-glass transformation in supercooled liquids (SCLs) is followed by the formation of intermediate metastable states in which a dramatic increase in viscosity. These states expose anomalous temperature behavior of transport characteristics commonly studied above the glass transformation temperature $T_{g}$, established by scanning calorimetry [1]. An intriguing aspect in glass transformation is the apparent connection between dynamics and thermodynamic features [2]. In particular, there is a great interest in complex, experimental and theoretical, studies of the temperature-temporal behavior of primary structural relaxation in SCLs [1], which is often similar to that in other glass formers [3-8].

Dynamic data on the relaxation timescale $\tau_{T}^{(\exp )}$ determined in viscoelastic, dielectric, conductivity, mechanical relaxation, light and neutron scattering experiments is regarded as one of the main keys to the understanding of mechanisms of the structural glass transformation $[1,9,10]$. A unified approach to the problem given within one coherent framework remains a challenge for theorists [11]. Here we put forward a mesoscopic-scale consideration of the primary relaxation observed during vitrification in liquids and solids and approached by percolative-geometric [12], dynamic-stochastic [8] and thermodynamic-statistic [13] treatments. In this study we focus on the mechanisms of the primary in solid and liquid glass forming materials specified by space-relaxation dimensions fractal clusters. We also stress the relationships between thermodynamic and dynamic observable quantities, which are raised from the underlying constraints imposed on degrees of freedom in glass formation systems. Similarly to the case of the universal equation established for characteristic temperatures [13], the universal mechanisms of relaxation will be found for distinct glass formers, presented here by SCLs, polymers, orientational quadrupolar-glass molecular, and spin-glass metallic and nonmetallic solids. The paper is organized as follows. In Sec. 2, the experimental and theoretical preliminaries are provided for dynamical macroscopic characteristics commonly describing non-Arrhenius tempera- 
ture and non-Debye temporal behavior in glass forming materials. Predictions for thermal expansion which are given in terms of the dynamical and thermodynamical (characteristic temperatures) parameters are provided in Sec. 3. The mechanisms of the primary relaxation near the glass transformation temperature are discussed in Sec. 4 for distinct glass formers, including a special case of the ortho-para- $\mathrm{H}_{2}$ mixtures. Conclusions are summarized in Sec. 5.

\section{Preliminary}

\subsection{Fitting forms}

The phenomenological Vogel-Fulcher-Tammann (VFT) fitting form, namely

$$
\tau_{T}^{(V F T)}=\tau_{\min } \exp \left(\frac{D_{g} T_{0}}{T-T_{0}}\right)
$$

is widely used to describe non-Arrhenius temperature behavior of the structural relaxation in amorphous liquids and solids; $D_{g}$ is the so-called strength index [14] and $T_{0}$ is the VFT temperature. Proposed in the 1920s [9], Eq. (1) performs well within the temperature range established as $T_{g} \leq T<T_{c}[13,15]$. Here $T_{c}$ is the crossover temperature $T_{c}$, which separates the moderately and strongly SCL states [13], distinguished in mode coupling theory (MCT) [1]. The pre-factor $\tau_{\min }^{(\exp )}=10^{-14 \pm 2} \mathrm{~s}$ reflects the Debye molecule vibrational times, characteristic of the normal-liquid state. Besides Eq. (1), the non-Debye time-decay of structural correlations is also a generic feature of collective relaxation dynamics in supercooled states of all glass formers. The late-time dynamical response function is commonly fitted through the phenomenological Kohlrausch-Williams-Watts (KWW) form by the two temperature-dependent parameters $\beta_{T}$ and $\tau_{T}$ [1]. Near $T_{g}$, the slow part of the relaxation function is due to the late-time asymptote

$$
\varphi_{S}^{(\exp )}(t, T) \propto \exp \left[-\left(\frac{t}{\tau_{T}}\right)^{\beta_{g}}\right], \text { for } t>>\tau_{g}
$$

Here $\beta_{g}$ is the KWW stretching exponent that commonly weakly depends on $T$ in the vicinity of $T_{g}$.

The process of glass formation is followed by the strengthening of dynamic correlations. As the temperature approaches $T_{g}$ from above, it evolves smoothly starting from the Debye behavior typical of the normal liquid state $[16,18,19]$. The relaxation function can be therefore approximated by the ensemble of modified Debye-type clusters. The trial ensemble of clusters of random radius-size $R$ and relaxation time $\tau_{D}(R)$ is characterized by the dynamically stable Debye clusters with the mean size
$R_{T}$ and relaxation time $\tau_{D T}$. Thus, $\varphi_{T}(t)$ gradually changes from the high- $T$ Debye form to that given as

$$
\varphi^{(\bmod )}(t, T)=\int_{0}^{\infty} \exp \left[-\frac{t}{\tau_{D}(R)}\right] P_{T}^{(\bmod )}\left(R / R_{T}\right) d R,
$$

where $P_{T}^{(\bmod )}\left(R / R_{T}\right) \equiv P(R, T)$ stands for the radius-size distribution function for relaxing structural units. The latter are solid-like clusters in the case of SCLs. Following the idea of the phase-ordering kinetics [20], the dynamic scaling law

$$
\tau_{D}\left(R / R_{T}\right)=\tau_{D T}\left(\frac{R}{R_{T}}\right)^{z_{g}} \text {, with } R_{T} \geq R_{a}
$$

was employed [12] near $T_{g}$, with the help of the dynamic cluster-dimension exponent $z_{T}$ for clusters restricted by the minimum cluster size $R_{a}$.

\subsection{Fractal cluster treatment}

A schematic scenario of the stretched-time relaxation can be figured out as a percolation process of fractal clusters of fixed structure, performing a diffusive motion at a given temperature $T$. The cluster-volume distribution density function $P(V, T)$ is thought of to be distinct for small-volume $\left(V<V_{T}\right)$ clusters and for large-volume $\left(V>V_{T}\right)$ clusters distinguished by the typical-cluster volume $V_{T}$. The radius-size cluster statistics of self-similar clusters is therefore described by $P_{T}\left(R / R_{T}\right) \equiv P(V, T) d V / d R$. The cluster fractal dimension $d_{f}$ is defined through [21]

$$
V=V_{T}\left(\frac{R}{R_{T}}\right)^{d_{f}} \text {. }
$$

In this way, the process of structural relaxation is treated as a percolation of correlated sites or bonds [21]. The site-percolation relaxation can be described through the model density probability [12]

$$
P_{T}^{(\bmod )}(x) \sim x^{u \tilde{d}-1} \exp \left(-x^{\widetilde{d}}\right), \quad x=\frac{R}{R_{T}}, u=\frac{d_{f}}{d_{s}} .
$$

It is introduced through the two cluster-shape parameters: $d_{f}(5)$ and $d_{s}$, which is the effective dimension of space of late-time relaxation attributed to large clusters competing with small clusters of size $R<R_{T}$.

In order to establish the model parameter $u$ for distinct glass-formers, we have performed the model-independent analysis of dielectric response data in SCLs proposed by in Ref. 16. The well known Dixon-Nagel universal master curve [16] scales the dielectric susceptibility spectrum over a wide temperature range above $T_{g}$ and over more than ten orders of magnitude in frequency $\omega$. In order to fit the Dixon-Nagel curve, and thereby to describe the experimental data on dielectric susceptibility, we estimated the model susceptibility 


$$
\chi_{T}^{(\bmod )}(\omega)=\chi_{T}(0) \int_{0}^{\infty} \frac{P_{T}^{(\bmod )}(x)}{1-i \omega \tau_{D}(x) d x}
$$

which is a Fourier analogue of Eq. $(3) ; \chi_{T}(0)$ is the static susceptibility. The extended analysis of the Dixon-Nagel master curve provided on the basis of the model distribution function $P_{T}^{(\bmod )}(6)$ and the Debye-cluster relaxation time $\tau_{D}(R)(4)$ is similar to that described in Fig. 1 in Ref. 12. The results [17]

$$
\begin{gathered}
u_{g}^{(\text {pol })}=0.33 \pm 0.03, u_{g}^{(\text {netw })}=0.26 \pm 0.04 \\
\text { and } u_{g}^{\text {liq }}=0.25 \pm 0.06
\end{gathered}
$$

obtained, respectively, for supercooled polymeric, network, and molecular liquids will be employed below for the cluster-dimension description in these materials.

Another simple-model estimation for the slow-relaxation function

$$
\varphi_{S}^{(\bmod )}(t, T) \sim \int_{0}^{\infty} \exp \left[-\psi_{\lambda}(x)\right] d \xi \propto \frac{\exp \left[-\psi_{\lambda}\left(x_{0}\right)\right]}{\sqrt{\psi_{\lambda}^{\prime \prime}\left(x_{0}\right)}},
$$

follows from Eq. (3). It is presented with the help of the auxiliary function $\psi_{\lambda}$, the variable $x=R / R_{T}$, and the temporal parameter $\lambda=t / \tau_{D T}$. Application of the standard method of steepness descent leads to the late-time asymp-

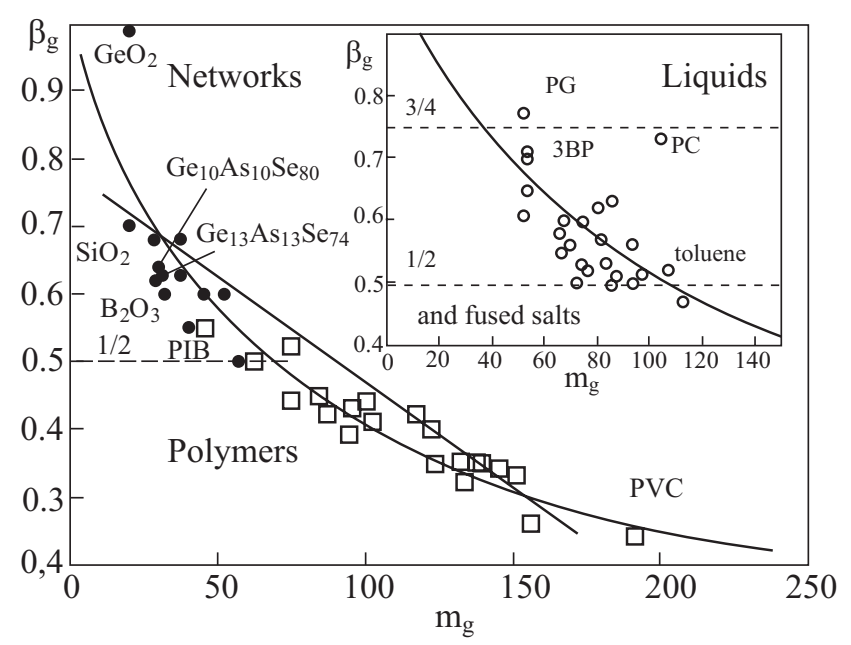

Fig. 1. Non-Debye against Non-Arrhenius behavior near $T_{g}$. Symbols are experimental data on dielectric, mechanical and light-scatterer relaxation, Ref. 14. Solid lines correspond to Eq. (15). The fitting parameters $m_{\beta}^{(\mathrm{pol})}=m_{\beta}^{(\text {netw })}=70 \pm 5$ are found for polymeric and network glass-forming liquids, and $m_{\beta}^{(\text {liq })}=100 \pm 10$ for simple, complex and alcoholic SCLs, extended by molten salts (shown in inset). The thick dashed line is given by the overall linear phenomenological fitting $m_{g}=250-320 \beta_{g}$, reported in Ref. 14, and the thin dashed lines indicate, approximately, the upper and lower limits of the data. tote, shown in Eq. (9) for $\lambda>>1$, where $\psi_{\lambda}^{\prime \prime}$ stands for the second-order derivative with respect to $x$. The saddle point $x_{0}=\left(\lambda z / d_{f}\right)^{1 /\left(z+d_{f}\right)}$ is established by the stationary condition $\left[\psi_{\lambda}^{\prime}\left(x_{0}\right)=0, \psi_{\lambda}^{\prime \prime}\left(x_{0}\right)<0\right]$ which is valid for late times $t>>\tau_{D T}\left(\left|d_{f}-1\right| / z \beta\right)^{1 / \beta}$. Then, through a comparison of Eq. (9) with the experimental data (2), one finds the model estimate for the stretching exponent resulting in the model relation [12]

$$
\beta_{g}^{(\bmod )}=\frac{d_{s}}{z_{g}+d_{s}} .
$$

When the effective dimension $d_{s}$ and the stretching exponent $\beta_{g}$ can be established independently, Eq. (10) provides the cluster-growth exponent prediction

$$
z_{g}^{(\bmod )}=d_{s}\left(\frac{1}{\beta_{g}}-1\right) .
$$

Also, a relation between the primary relaxation time and the mean intrinsic time of solid-like Debye clusters:

$$
\tau_{T}=\tau_{D T} \beta_{g}\left(1-\beta_{g}\right)^{1 / \beta_{g}-1}
$$

follows from the simplest model (9), where $\tau_{T}$ and $\beta_{g}$ can be observed by means of the KWW fitting form.

Using $\tau_{D T}=\tau_{a}\left(R_{T} / R_{a}\right)^{z_{g}}$ in the scaling form (4) and taking into account Eq. (12), the timescale steepness at $T_{g}$ (fragility) estimates as

$$
\begin{gathered}
m_{g}=-\left(\frac{d \lg \tau_{T}}{d \ln T}\right)_{T=T_{g}}=m_{z}^{*} z_{g} \text { with } \\
m_{z}^{*}=-\left(\frac{d \lg R_{T}}{d \ln T}\right)_{T=T_{g}} \cdot
\end{gathered}
$$

This provides a link between the fragility $m_{g}$ and the cluster-dimension dynamic exponent $z_{g}(11)$, namely [8]

$$
m_{g}=m_{\beta}^{*}\left(\frac{1}{\beta_{g}}-1\right) \text {, with } m_{\beta}^{*}=m_{z}^{*} d_{s} .
$$

This can be read as an alternative to Eq. (10) prediction for the stretching exponent

$$
\beta_{g}=\frac{m_{\beta}^{*}}{m_{g}+m_{\beta}^{*}} .
$$

The experimental data on structural relaxation shown in Fig. 1 provide evidence for $m_{z}^{*}>0$ (13), i.e., the growth of correlations in collective dynamics under cooling. This view is based on assumption of that correlated regions of finite size $R_{T}$ and of a certain structure exist, for which $d R_{T} / d T<0$, near $T_{g}$, ensues a stabilization of the material-weakly-dependent parameter $m_{z}^{*}$. Such a kind of 
model-independent clusters is observed indirectly in Fig. 1, through the parameter $m_{\beta}^{*}$, establishing the effective dimension $d_{s}(14)$ derived below.

\section{Link to thermal expansion}

Let us re-present the dynamic scaling law (4) in the form $R_{T} \sim R_{a}\left(\tau_{D T} / \tau_{a}\right)^{1 / z_{g}}$. When the VFT Eq. (1) is additionally used for $\tau_{T}$ in Eq. (12), the cluster mean size

$$
R_{T}^{(V F T)}=R_{a} \exp \left(\frac{D_{g}}{z_{g} \varepsilon_{T}}\right), \quad T_{g} \leq T<T_{c}
$$

is made explicit. Furthermore, if for the fractal clusters (5) one introduces the thermal expansion coefficient

$$
\alpha_{T} \equiv \frac{1}{V} \frac{d V}{d T}
$$

the estimate

$$
\alpha_{T}^{(V F T)}=-\frac{d_{f} \ln 10}{z_{g}} \frac{m_{g}^{* 2}}{m_{g}-m_{g}^{*}} \frac{T_{0}}{\left(T-T_{0}\right)^{2}}, \quad T_{g} \leq T<T_{c}
$$

immediately follows from Eqs. (17) and (16). The strongly material-dependent expansion coefficient (18) is presented with the help of the known relation $D_{g}=m_{g}^{* 2}\left(m_{g}-m_{g}^{*}\right)^{-1} \ln 10$, earlier established [14] for the VFT phenomenological form. Then, the material-independent quantity

$$
\left|\alpha_{g}\right| T_{g}=m_{z}^{*} d_{f} \ln 10
$$

can be readily established for fractal clusters. It is deduced from Eqs. (17) and (13), taken at $T_{g}$, without recourse to any fitting form, including the VFT case (16). Moreover, one can examine that Eqs. (18) and (19) are self-consistent. In addition, the model equation

$$
\left|\alpha_{g}^{(\bmod )}\right| T_{g}=m_{\beta}^{*} u \ln 10
$$

can be obtained, if the material-independent relations $m_{z}^{*}=m_{\beta}^{*} / d_{s}(14)$ and $u=d_{f} / d_{s}(8)$ are employed.

A direct observation of the cluster-dimension growth (11) through Eq. (13) becomes possible thanks to the neutron scattering data $[22,23]$ on the dynamical exponent $z_{g}$ available for supercooled polymers and analyzed in Ref. 8. Combining the result $m_{z}^{(\mathrm{pol})}=22 \pm 2$, obtained through analysis given in Fig. 2, with the output of dielectric and mechanical dynamical experiments for $m_{\beta}^{(\mathrm{pol})}=$ $=70 \pm 5$, derived in Fig. 1 , one finds $d_{s}^{(\mathrm{pol})}=3.2 \pm 0.3$, for the effective relaxation-space dimension (6).

Furthermore, taking into consideration the fact of the applicability of the model finding $u_{g}^{(\mathrm{pol})}=0.33 \pm 0.03$ (8), the fractal dimension (6) $d_{f}^{(\mathrm{pol})}=1.00 \pm 0.09$ is derived

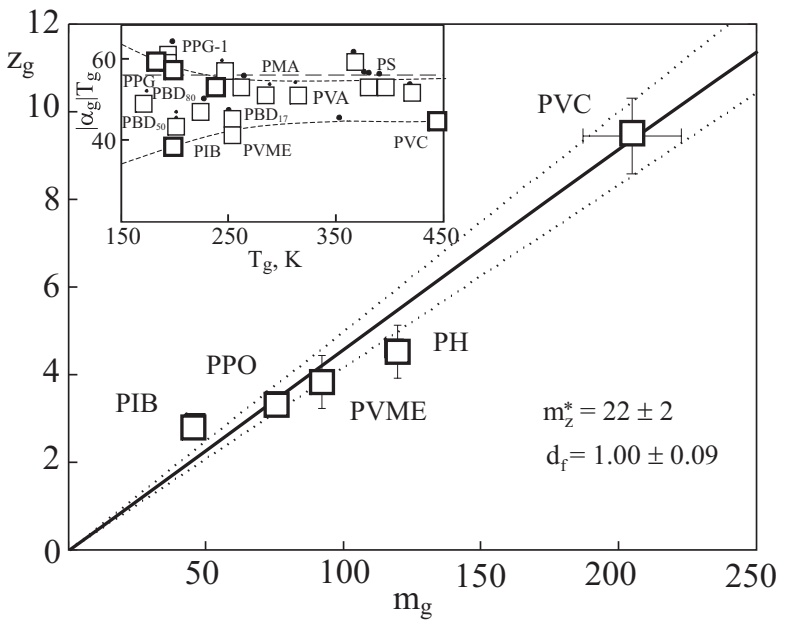

Fig. 2. Cluster-dimension dynamical exponent against fragility. The points are the quasi-elastic neutron scattering and fragility data reported for polymers in [22-24]. The solid, upper and lower pointed lines are drawn through Eq. (13) with $m_{z}^{*}=22,20$ and 24, respectively. Insert: model prediction for the expansivity relation in polymers against glass transformation temperature $T_{g}$. The thin and thick squares are the experimental data reported in, respectively, [14,22-24], re-estimated through Eq. (21) with $u_{g}^{(\exp )}=0.33$. Lines are guide to the eye.

here for tangled-chained structures common for glass forming polymers. Moreover, a model prediction

$$
\left|\alpha_{g}^{(\text {pred })}\right| T_{g}=u_{g}^{(\exp )} \ln 10 \frac{m_{g}}{1 / \beta_{g}-1}
$$

is obtained through Eqs. (20) and (14). With the aim of testing the material dependence of the model quantity described in Eq. (20), the model-equivalent relation (21) is plotted in the inset in Fig. 2. When one uses the well-known relation for the characteristic temperatures (see, e.g., Eq. (6) in Ref. 13)

$$
\frac{T_{g}}{T_{0}}=\frac{m_{g}}{m_{g}-m_{g}^{*}}
$$

a new prediction follows from Eq. (21), namely

$$
\left|\alpha_{g}^{(\text {pred })}\right| T_{0}=u_{g}^{(\exp )} \ln 10 \frac{\left(m_{g}-m_{g}^{*}\right)}{1 / \beta_{g}-1} .
$$

This can be compared with the model-equivalent relation

$$
\left|\alpha_{g}^{(\text {pred })}\right| T_{0}=u_{g}^{(\exp )} m_{\beta}^{*} \ln 10\left(1-\frac{m_{g}^{*}}{m_{g}}\right),
$$

which is smoothed through the parameter $m_{\beta}^{*}$, obtained above in Fig. 1. Finally, our findings for the cluster-shape parameter $u_{g}^{(\exp )}(8)$ enables one to provide analysis for all studied glass formers, presented in Fig. 3. 
As seen in Fig. 3, the predicted expansion coefficient $\alpha_{g}$ is expected to be strongly material dependent. Conversely, the quantity $\left|\alpha_{g}^{\text {(pred) }}\right| T_{0}$ changes weakly with chemical structure in glass forming liquids and polymers. This allows to find the minimum expansivity as $\alpha_{g}^{(\min )}=-(54 \pm 5) / T_{0}$, obtained in the fragile-glass limit $\left(m_{g} / m_{g}^{*} \rightarrow \infty\right)$.

\section{Cluster relaxation mechanisms}

The mechanisms of structural relaxation in glass forming polymers and structural disordered orientational-glass formers were discussed respectively in Refs. 25 and 8. In both cases the directed by random walk (DRW) mechanism, known from the restricted-diffusion models for $d_{s}=1$ extended over the dimension $d_{s}=3$, was established. The DRW model prediction is [8]

$$
z_{g}^{(D R W)}=3\left(\frac{1}{\beta_{g}}-1\right),
$$

that can be compared with Eq. (11). It seems reasonable to extend this mechanism to the site-disordered solid $o-p-\mathrm{H}_{2}$ $(\mathrm{OPH})$ mixtures, as the quadrupolar-glass $(\mathrm{QG})$ former,
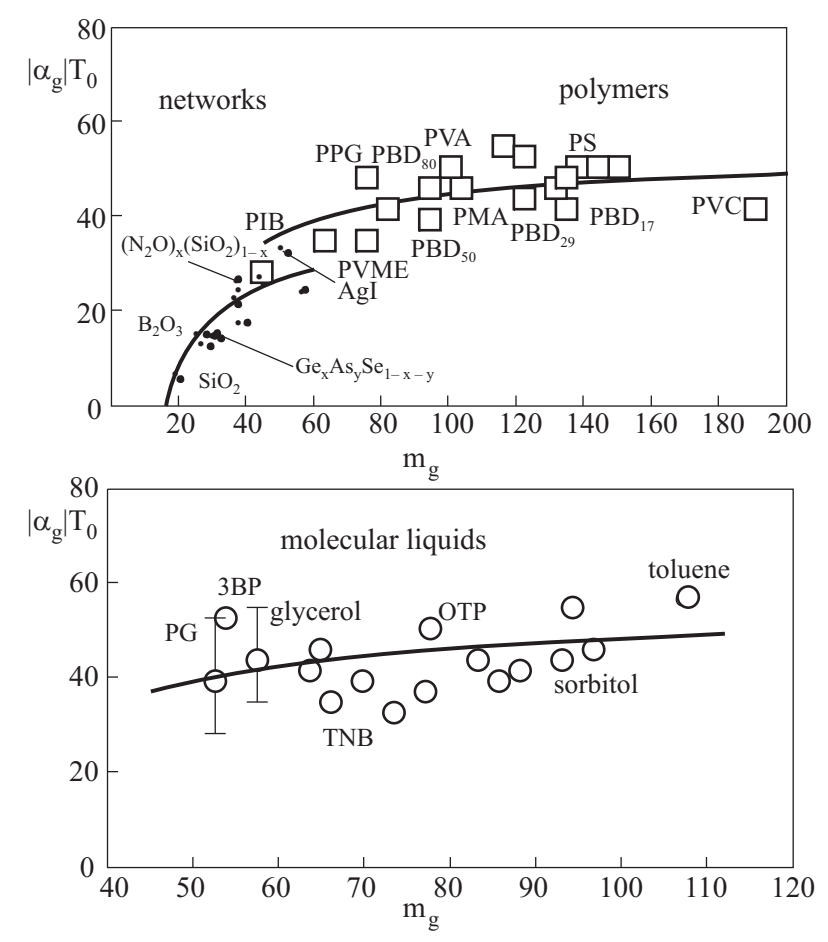

Fig. 3. Model predictions for the expansivity relations in different glass formers against fragility. Symbols and lines correspond to those shown in Fig. 1. The points are dynamic relaxation data [14] re-estimated through Eq. (23), with $u_{g}^{(\exp )}=0.33,0.26$ and 0.25 for, respectively, polymers (open squares), networks (closed circles), and molecular liquids (open circles). The solid lines are drawn through Eq. (24), with $m_{\beta}^{*}=65.70$ and 100 for, respectively, networks, polymers, and molecular (complex, simple, and alcoholic) liquids. though no dielectric loss data can be available [26]. In what follows, we seek to provide the data on the standard set of VFT and KWW dynamical exponents through the coarse-graining of microscopic model descriptions of OPH mixtures given in Refs. 27 and 28.

\subsection{The case of $\mathrm{OPH}$}

Microscopic treatment of the QG state in real OPH mixtures is based on the random-bond and random-site quadrupolar Hamiltonian introduced from the first principles $[26,29]$. The microscopic theory was developed in Ref. 27 within the framework of Bogolyubov's variational scheme developed in terms of the two local-order dynamical variables $\sigma_{i}$ and $\eta_{i}$. A description of the metastable rotational state, arising from the quenched site-substitutional disorder, is introduced through a set of macroscopic order parameters

$$
\begin{gathered}
q_{T}(x)=<\sigma_{i}^{2}+\eta_{i}^{2}>_{C}, p_{T}(x)=<\sigma_{i}^{2}-\eta_{i}^{2}>_{C}, \text { and } \\
\sigma_{T}(x)=<\sigma_{i}>_{C},
\end{gathered}
$$

given at a fixed temperature $T$ and a rotor-molecule concentration $x$ corresponding to the ortho-hydrogen overtion. Symbol $C$ denotes a configurational average over random realizations. The microscopic treatment provides a closed system for the order-parameter equations [27]. Their analysis at high temperatures indicates that the dynamical freezing into the short-ranged, bond-bond correlated quantum QG state occurs at a certain glass freezing temperature $T_{f}(x)=T_{g}(x)$ established in both thermodynamic and dynamic NMR experiments [26]. Below $T_{g}$, the isotropic (IQG, $q_{T}>>\sigma_{T} \gg p_{T}$ ) and anisotropic $\left(q_{T} \geq p_{T}>>\sigma_{T}\right)$ quadrupolar glass states are possible [27].

Within context of the theory of diluted magnetics, the outcomes of the QG theory are described through the two competing parameters

$$
\begin{gathered}
J(x)=<J_{i j}^{2}>_{C}^{1 / 2} \text { and } \\
\kappa_{T}(x)=\frac{<\left(h_{i}-h_{1}\right)^{2}>_{C}}{<h_{i}>_{C}}=\frac{h_{2}(x, T)}{h_{1}(x, T)},
\end{gathered}
$$

which are, respectively, the variance of the random exchange $J_{i j}$ and the ratio of the variance $h_{2}$ and mean $h_{1}$ for the fluctuation field $h_{i}$, both are the energetic parameters of the OPH Hamiltonian [27]. The IQG ground state $\left(q_{0}=q_{0}(x)<1, \sigma_{0}=0, q_{0}>>p_{0}\right)$ defined [30] through the QG order parameter $q_{T}(x)$ estimated at $T=0$ reads as

$$
\begin{gathered}
q_{0}(x)=q_{\max } \frac{1-\kappa_{0}^{2}(x)}{2}\left(1+\sqrt{1-\frac{3}{4 q_{\max }} \frac{\kappa_{0}^{2}(x)}{\left[1-\kappa_{0}^{2}(x)\right]^{2}}}\right), \\
\kappa_{0}(x)=1.18 \sqrt{\frac{x_{\max }-x}{n_{a} x}},
\end{gathered}
$$


where the parameter $q_{\max }$ establishes a certain scale of variation of $q_{0}$ at the maximum concentration $x_{\max }$; $n_{a}=12$ is the number of nearest neighbors in HCP lattice. The ground-state prediction (28) was carefully tested by the experimental data (see Fig. 1 in Ref. 30 and Fig. 3 in Ref. 28). The low- $T$ asymptotic behavior [27]

$$
\begin{gathered}
q_{T}(x)=q_{0}(x)-q_{02}(x)\left(\frac{T}{J(x)}\right)^{2}+q_{04}\left(\frac{T}{J(x)}\right)^{4} \text {, with } \\
J(x)=\Gamma \sqrt{n_{a} x},
\end{gathered}
$$

is presented by the Taylor series and the quadrupolar coupling constant $\Gamma=0.82 \mathrm{~K}$.

Fractal clusters. Following Ref. 30, $q_{0}(x)(28)$ can be improved by including distant rotor neighbors $n_{0}(x)$. In this way, a fractal structure of the IQG cluster is introduced here through the relations

$$
\begin{gathered}
n_{0}(x)=n_{a}\left(\frac{R_{0}}{R_{a}}\right)^{d_{f}}, R_{0}(x)=R_{a}\left(\frac{x}{x_{a}}\right)^{1 / d} \text {, for } \\
d>d_{f}, x \geq x_{a},
\end{gathered}
$$

where the fractal dimension $d_{f}$ which is less of the spatial dimension $d=3 ; R_{0}$ plays the role of the random-walk cluster correlation length, which exceeds the nearest-neighbor distance $R_{a}$. In the approximation of continuos medium, $x_{a}$ was estimated [30] as $3 / 4 \pi \sqrt{2} \approx 0.17$ corresponding to the observed lower-bound critical concentration $x_{\min }^{(\exp )} \approx 0.1$. Taking into account the upper-boundary data [26] $x_{\max }^{(\exp )}=0.55$, the modified structural-disorder parameter

$$
\begin{gathered}
\widetilde{\kappa}_{0}(x)=1.18 \sqrt{\frac{x_{\max }-x}{n_{0} x}}, \\
n_{0}(x)=n_{a}\left(\frac{x}{x_{a}}\right)^{d_{f} / d}, x_{\max }=0.55
\end{gathered}
$$

is employed to fit the data [31] on $q_{0}^{(\exp )}(x)$ through the modified Eq. (28). As the result, the fractal dimension $d_{f}=2.5 \pm 0.3$ is derived via the fitting analysis (shown below in Fig. 4). Remarkably, the given coarse-grained QG description is consistent with the model-independent critical dimension $d_{f}=5 / 2$, known for $d=3$ in the general percolation theory (see, e.g., Table III in Ref. 21). We therefore put $d_{s}=d=3$ in Eq. (11) that justifies the application of Eq. (25) to the case of OPH.

Characteristic temperatures. The orientational-order freezing mechanism rationalized [32] in terms of the short-range ordering standard molecular field competing with strongly correlated intrinsic fluctuation field. In this way, $T_{g}(x)$ is treated as a molecular-fluctuation crossover field temperature. In the weakly rotor-correlated para-rotational phase (PR, $T>T_{g}$ ), a formation of the moderately supercooled state was shown [32] to be driven by

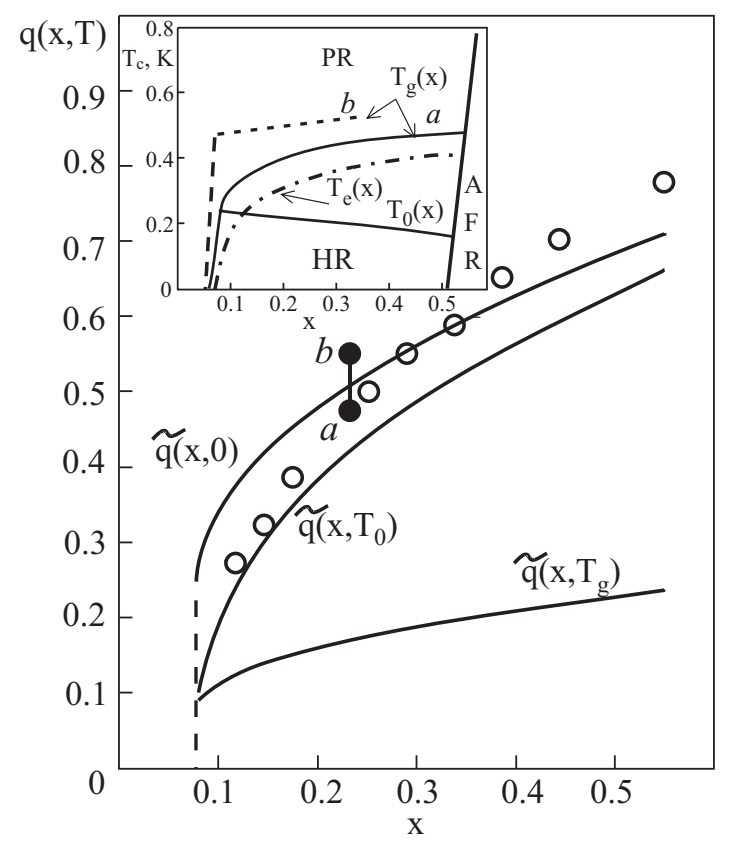

Fig. 4. The quadrupolar-glass orientational-order parameter in OPH molecular mixtures against of ortho-hydrogen concentration at distinct temperatures. The open circles correspond to the linear extrapolation to $T=0$, after Meyer and Washburn [31]. The closed circles are given by the linear $(a)$ and non-linear $(b)$ extrapolations, after Sullivan and co-workers [33]. Lines: $\widetilde{q}(x, 0)$ is the extrapolated QG ground state $\widetilde{q}_{0}(x)$ described in Eqs. (28) and (31), with $d_{f}=2.5$ and $q_{M}=0.71$. The data $\widetilde{q}\left(x, T_{0}\right)$ and $\widetilde{q}\left(x, T_{g}\right)$ are Eqs.(29), modified through Eq. (31), shown at the characteristic temperatures $T_{g}^{(a)}(x)(32)$ and $T_{0}(x)$ (36). Inset: the quadrupolar-glass transformation characteristic temperatures against concentration. The solid lines are $T_{g}^{(a)}(x)$ and $T_{0}(x)$. The dashed-dotted line sketches the ergodic-instability temperature [17]. Notations: PR-para-rotational short-range ordered (supercooled) phase, AFR - antiferro-rotational long-range ordered phase, HR — hindered rotor phase [34].

the random Zeeman-type field [26]. Below $T_{g}$, the Zeeman-field effects are suppressed by the by reaction Onsager field [27,30], which, along with the molecular exchange-coupling field, determines the formation of QG-type clusters.

A microscopic-level description of the so-called PR-QG boundary [26,30], here determined as $T_{g}(x)$, remains an unsolved problem. Nevertheless, a peculiarity in the temperature behavior of $q_{T}(x)$ near $T_{g}$ can be expected, when the PR-QG boundary is introduced within the framework of the coarse-grained description $\widetilde{q}_{T}(x)$, given through Eqs. (29), (28), and (31), as a crossover between the moderately supercooled state $\left(\widetilde{q}_{g}>\sigma_{g}\right)$ and the strongly moderately supercooled state $\left(\widetilde{q}_{g}=\sigma_{g}^{2}\right)$. In Ref. 32, this peculiarity was discussed through the two approximate schemes called by the «intrinsic-field self-compensation effect» near $T_{g}(x)$. The first scheme 
treats the crossover point as $x$-independent kink observed for $\widetilde{q}_{T}(x)=\widetilde{q}_{0}(x) / 3$ at the temperature

$$
T_{g}^{(a)}(x)=J \sqrt{\frac{2 \widetilde{q}_{0}}{3 \widetilde{q}_{02}}}
$$

where the random-mean-square exchange energy $J(x)$ is defined in Eq. (27) and specified in Eq. (29). An alternative approach considers the crossover line as an inflection point $\partial^{2} \widetilde{q}_{T}(x) / \partial T^{2}=0$ observed at the temperature

$$
T_{g}^{(b)}(x)=J \sqrt{\frac{\widetilde{q}_{02}}{\widetilde{q}_{04}}} .
$$

Numerical analysis of the proposed cluster description in $\mathrm{OPH}$ is given in Fig. 4.

Thermodynamic instability. Within the mesoscopic QG-cluster treatment of orientationally-correlated rotors, given in the concentration domain $x_{\min }<x<x_{\max }$, the corresponding correlation length $R_{0}$ is constrained by $R_{a} \lesssim R_{0} \lesssim 3 R_{a}$. This implies that the distant rotors with $R>3 R_{a}$ are almost isolated relaxing units. Correspondingly, the rotational heat capacity $C_{\text {rot }}$ attributed to the rotational degrees of freedom of the system is commonly due to the contributions from the strongly correlated (collective) rotational excitations $\left(C_{\text {cor }}\right)$ and from the hindered rotation of weakly correlated rotors $\left(C_{\text {hind }}\right)$, namely

$$
C_{\text {rot }}=C_{\text {cor }}+C_{\text {hind }} \text {. }
$$

The last term was described [32] through the Schottky-type anomaly of an isolated rotor modified by distant rotors. Similarly to the order parameter $q_{T}(x)$, the microscopic theory suggests the low- $T$ asymptote for $C_{\text {cor }}(x, T)$ represented here as

$$
\frac{C_{\text {cor }}(x, T)}{x R}=2 s_{0}(x)\left[\left(\frac{T}{T_{0}(x)}\right)^{2}-1\right],
$$

with $R$ is the gas constant, $T_{0}(x)=J(x) \sqrt{s_{0}(x) / 3 s_{02}(x)}$ and the parameters $s_{0}(x)$ and $s_{02}(x)$ are given in Eq. (20) in Ref. 27. The thermodynamic-instability in Eq. (35) establishes the VFT temperature

$$
T_{0}(x)=\Gamma \sqrt{\frac{n_{0} x}{6}}\left(1-\widetilde{q}_{0}\right) \sqrt{1-\widetilde{\kappa}_{0}^{2}-\frac{\widetilde{\kappa}_{0}^{2}}{8 \widetilde{q}_{0}} \frac{\left(1-2 \widetilde{q}_{0}\right)}{\left(1-\widetilde{q}_{0}\right)}}
$$

given through the functions $\widetilde{q}_{0}(x)$ and $\widetilde{\kappa}_{0}(x), n_{0}(x)$ shown in Eqs. (28) and (31), respectively, and is plotted in the inset in Fig. 4.

Fragility and stretching exponent. In the absence of the loss dielectric data, the non-Debye primary relaxation in OPH mixtures could be derived from the order-parameter temporal behavior observed in the NMR spectra [26]. The expected VFT-type behavior, introduced here through $T_{0}(x)$ and $T_{g}(x)$ suggests the OPH fragility

$$
m_{g}(x)=m_{g}^{*}\left[1-\frac{T_{0}(x)}{T_{g}(x)}\right]^{-1},
$$

defined with the help of Eq. (22). This finding provides the desired stretching exponent of the KWW form (2), namely

$$
\beta_{g}(x)=\frac{m_{\beta}^{*}}{m_{g}(x)+m_{\beta}^{*}}
$$

when Eq. (15) is additionally employed. Numerically, these predictions are analyzed in Fig. 5.

In Fig. 5, both the versions discussed in Eqs. (36) and (32) are drawn as two ways of the order-parameter zero-temperature extrapolation. The numerical discrepancy between the two versions, estimated through the experimental data [33], is shown by the error bar $a b$ in Fig. 4 .

\subsection{Other glass formers}

In Fig. 6 two distinct mechanisms are suggested for site-disordered and mixed crystals and also for site-ordered «bond-frustrated» plastic crystals, all characterized in Table 1 in Ref. 8, now extended by OPH mixtures. The first type of materials is described by the DRW model in
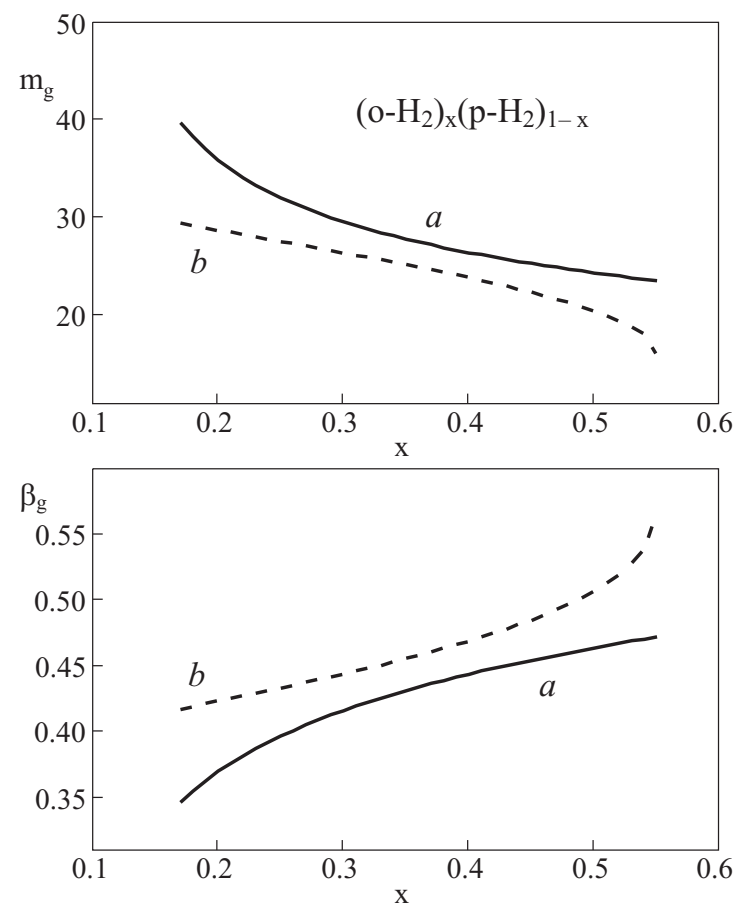

Fig. 5. Primary relaxation characteristics of $\mathrm{OPH}$ mixtures against ortho-hydrogen concentration: the fragility (upper plot) and stretching exponent (lower plot). The solid lines are driven through Eqs. (37) and (38) with $m_{g}^{*}=15$ for the fragility and $m_{\beta}^{*}=21$ for the stretching exponent, common for site-disordered crystals [8], and $T_{0}(x)$ and $T_{g}^{(a)}(x)$ are given in Eqs. (36) and (32) with the parameters found in Fig. 4. The dashed lines correspond to the case $T_{g}^{(b)}(x)(33)$. 


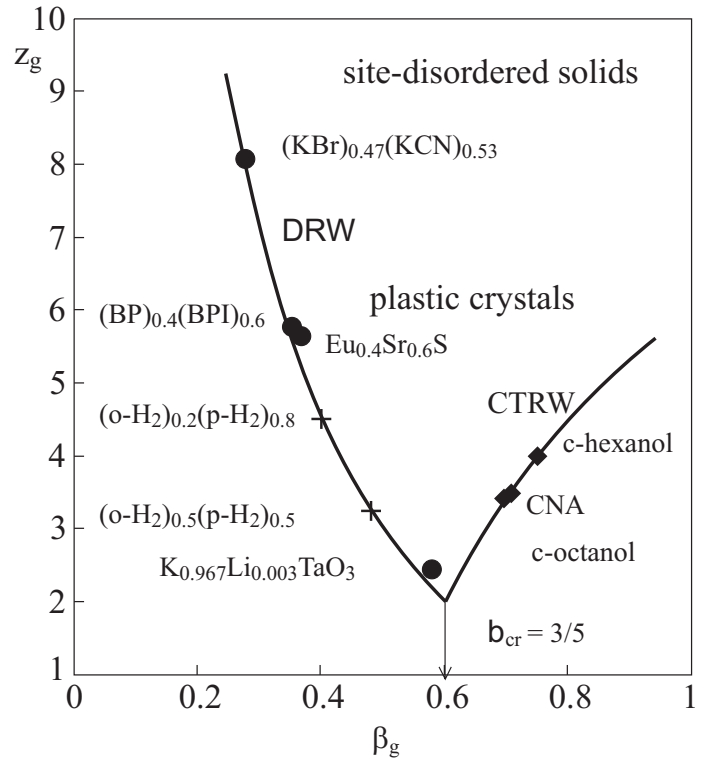

Fig. 6. Theoretical predictions for the primary relaxation mechanisms in orientational glasses: diffusion exponent against stretching exponent. The solid lines are given by Eqs. (25) and (39). Symbols (circles and squares) are drawn through the same equations and shown for materials with the known data on $\beta_{g}^{(\exp )}$ [14]. The crosses correspond to the OPH predictions on $\beta_{g}^{(\text {pred })}$ found in Fig. 5.

Eq. (25). Remarkably, that the same relaxation mechanism with $d_{s}=3$ is attributed to polymers [8] and also to non-polymeric SCLs, for which though $d_{s}=6$ was found [13].

Although Eq. (14) was analyzed in Ref. 8 and $m_{\beta}^{(P C)}=60$ was found for plastic crystals, the relaxation mechanism was not identified. Adopting the Brownian diffusion $\left.\left(z_{\mathrm{cr}}=2\right)\right)$ as the critical regime for any subdiffusion dynamics $\left(\beta_{\mathrm{cr}}=3 / 5\right.$ shown in Fig. 5$)$, the dynamic exponent (11) for plastic crystals

$$
z_{g}^{(C T R W)}=6\left(2-\frac{1}{\beta_{g}}\right) \text { and } \beta_{g} \geq \frac{3}{5},
$$

with $d_{g}^{(P C)}=6$ (along with $m_{z}^{(P C)}=10$ ) fits well the experimentally observed data. Even though the suggested relaxation regime in known [] only in the effective space $d^{(C T R W)}=1$ of the Continues Time Random Walk (CTRW) model, as shown in Eq. (85) in Ref. 17, Eq. (39) extends this mechanism over $d^{(\text {CTRW })}=6$.

\section{Summary}

We have seen that from the macroscopic point of view no conceptual gap exists between the supercooled states in metallic and non-metallic spin glasses, dipolar and quadrupolar orientational glasses, and molecular and polymeric structural glasses. A fruitful analogy between all three fields is widely explored by many researchers [3-7] that challenges the development of a generalized theoretical consideration. In our study, a cooperative process of glass formation it treated in terms of material-abstract relaxing units, whose relaxation dynamics is driven by late-time correlations associated with large clusters. It is shown that the universal (material-independent) features of the $\alpha$-relaxation under cooling are stipulated by the slow growing of correlations as well as by self-similarity of the mesoscopic-scale hierarchical structure of these correlations. Though a specification of correlations depends on the chosen theoretical scheme, their structure similarity is evidently manifested through the existence of weakly material-dependent parameters, which in turn provide a link between the dynamic exponents and thermodynamic parameters of glass formers. As additionally shown in Ref. 17, the large clusters attributed to the late-time spatial correlations and described here through the KWW asymptotic scaling form are self-consistent with small clusters, revealed in turn through the short-time von Schweidler scaling form. As the results, this additionally ensures the existence of the wide intermediate scale implicit in the universal Dixon-Nagel curve. Within this context, the typical cluster radius size $R_{T}$ emerges as the upper and lower bound for self-similar asymptotically small and large clusters.

It has been earlier argued [8] that regardless of underlying microscopic realizations in distinct materials, the structural relaxation is driven by local random fields in glass formers with structural disorder (including polymers) can be described on the mesoscopic-scale level by DRW model with $d_{s}=3$. Although the QG is the first representative of orientational glass in site-disordered crystals found by Sullivan's group through the NMR spectroscopy [26], the macroscopic parameters of the standard VFT and KWW forms were not yet established. In a certain sense, we fill this gap making predictions in Fig. 5. Moreover, it is shown that the QG clusters in OPH mixtures are of fractal dimension $d_{f}=5 / 2$ and they relax in space $d_{f}=5 / 2$, similar to all other site-disordered glass forming materials. Remarkably, that the same relaxation mechanism is established for all simple, complex, and alcoholic liquids, though in this case $d_{s}=6$ and $d_{f}=3$ [13]. In contrast, the orientational-order relaxation in site-ordered plastic crystals is suggested to be driven according to the CTRW model treated in space with $d_{s}=6$. Finally, the found expansion thermal expansion at $T_{g}$ challenges new experimental research in SCLs, polymers and networks.

Acknowledgments. The financial support by $\mathrm{CNPq}$ is acknowledged.

1. W. Götze and L. Sjögen, Rep. Prog. Phys. 55, 241 (1992).

2. P.G. Debenedetti and F.H. Stillinger, Nature 410, 259 (2001)

3. C.A. Angell, Science 267, 1924 (1995).

4. J. Souletie, J. Phys. France 51, 883 (1990). 
5. R.V. Chamberlin, Phys. Rev. Lett. 82, 2520 (1999).

6. M. Mézard and G. Parisi, Phys. Rev. Lett. 82, 747 (1999).

7. R.H. Colby, Phys. Rev. E61, 1783 (2000).

8. V.B. Kokshenev and N.S. Sullivan, J. Low Temp. Phys. 122, 221 (2001).

9. C.A. Angell, K.L. Ngai, G.B. McKenna, P.F. McMillan, and S.W. Martin, J. Appl. Phys. 88, 3113 (2000).

10. K. Binder, J. Baschnagel, and W. Paul, Prog. Polym. Sci. 28, 115 (2003).

11. K. Binder, J. Baschnagel, and W. Paul, Prog. Polym. Sci. 28,115 (2003).

12. V.B. Kokshenev, Phys. Rev. E57, 1187 (1998).

13. V.B. Kokshenev, P.D. Borges, and N S. Sullivan, J. Chem. Phys. 122, 114510 (2005).

14. R. Böhmer, K.L. Ngai, C.A. Angell, and D.J. Plazek, J. Chem. Phys. 99, 4201 (1993).

15. R. Richert and C.A. Angell, J. Chem. Phys. 108, 9016 (1998).

16. P.K. Dixon, L. Wu, S.R. Nagel, B.D. Williams, and J.P. Carini, Phys. Rev. Lett. 65, 1108 (1990).

17. V.B. Kokshenev, in: Atomic and Molecular Cluster Research, Y.L. Ping (ed.), Nova Science Publishers Inc., N.Y. (2006).

18.A. Hunt, J. Phys. Condens. Matter 6, 8087 (1994).

19. R.V. Chamberlin, Europhys Lett. 33, 545 (1996).
20. J. Bray, Adv. Phys. 43, 357 (1994).

21. M.B. Isichenko, Rev. Mod. Phys. 64, 961(1992).

22. J. Colmenero, A. Alegria, A. Arbe, and B. Frick, Phys. Rev. Lett. 69, 478 (1992).

23. J. Colmenero, Physica A201, 38 (1993).

24. K.L. Ngai, in: Disorder Effects on Relaxation Processes: Glasses, Polymers, Proteins, R. Richert, and A. Blumen (eds.), Springer, Berlin (1994).

25. V.B. Kokshenev and N.S. Sullivan, Phys. Lett. A208, 97 (2001).

26. B. Harris and H. Meyer, Canad. J. Phys. 63, 3 (1985).

27. V.B. Kokshenev, J. Low Temp.Phys. 104, 1 (1996).

28. V.B. Kokshenev, J. Low Temp. Phys. 104, 25 (1996).

29. V.B. Kokshenev, Solid State Commun. 55, 143 (1985); V.B. Kokshenev and A.A. Litvin, Fiz. Nizk. Temp. 13, 339 (1987) [Sov. J. Low. Temp. Phys. 13, 195 (1987)].

30. V.B. Kokshenev, Phys. Rev. B53, 2191 (1996).

31. H. Meyer and S. Washburn, J. Low Temp. Phys. 57, 31 (1984).

32. V.B. Kokshenev, J. Low Temp. Phys. 111, 489 (1998).

33. D. Zhou, C.M. Edwards, and N.S. Sullivan, Solid State Commun. 60, 901 (1986).

34. K. Kim and N.S. Sullivan, J. Low Temp. Phys. 114, 173 (1999). 